\title{
Netrin-1 signaling mediates NO-induced glial precursor migration and accumulation
}

Cell Research (2010) 20:238-241. doi: 10.1038/cr.2010.7; published online 19 January 2010

\section{Dear Editor,}

In the central nervous system, cell migration and accumulation occur widely to construct a complex neural network during mammalian development [1]. Previous studies reported that both the gaseous messenger molecule nitric oxide (NO) [2-4] and widely studied axon guidance/migration cues such as Netrins $[5,6]$ regulated the migratory behavior of cells in the nervous system. However, little is known about whether there is any crosstalk between NO and guidance/migration cues during neural cell migration and accumulation. Here, we report novel evidence indicating that $\mathrm{NO}$ induces glial precursor (GP) cell migration and accumulation in vitro, and that this process is mediated by Netrin-1 signaling.

In preliminary tests, A2B5 ${ }^{+}$GPs (Figure 1Ai) derived from rhesus embryonic stem (ES) cells were found to give different responses to sodium nitroprusside (SNP, an NO donor, 0-6.25 $\times 10^{3} \mu \mathrm{M}$ ), including differentiation, motility, and apoptosis/necrosis (not shown). Interestingly, the cells were able to migrate and accumulate when stimulated by 10-250 $\mu \mathrm{M}$ SNP (Figure 1Aii-iv). In such conditions, GPs gradually extended neurites (Supplementary information, Movies S1, S2) with active growth cone-like structures at the tip where abundant $\beta$-actin was organized (Figure 1Aii, $24 \mathrm{~h}$ ), polarized, migrated in chains (Figure 1Aiii, 36 h; Supplementary information, Movie S3), and eventually accumulated into gliospheres (Figure 1Aiv, $72 \mathrm{~h}$ ). The migratory behavior of GPs was not observed in regular expansion culture conditions without SNP treatment (Supplementary information, Movie S4) or with treatment by photodegraded SNP (Supplementary information, Figure S1). Further analysis indicated that the number of gliospheres accumulated was related to the SNP concentration in a dose-dependent manner (Figure 1Av). In order to verify the existence of NO released from SNP, $\mathrm{NO}_{2}^{-}$in the medium was examined by the Griess method [7]. The results confirmed that there was prolonged $\mathrm{NO}_{2}^{-}$generation after SNP addition (Figure 1Avi), implying continuous generation of NO from exogenous SNP during cell migration and accumulation. Interestingly, more $\mathrm{NO}_{2}^{-}$was detected in the group comprising GPs treated with SNP than in the group with SNP in the absence of GPs (Figure 1Avi), indicating that additional endogenous NO was synthesized within the cells.

To test whether the NO-induced cell migration and accumulation could be mediated by axon guidance/migration cues, we first analyzed the expression profile of Netrins in the GPs before and after SNP treatment (Figure $1 \mathrm{Bi}-\mathrm{v})$ because Netrins were reported to be expressed in glial lineage cells $[8,9]$. The semi-quantitative RT-PCR and immunocytochemistry results indicated that both Netrin-1 and Netrin-4 were up-regulated in the GPs after SNP treatment (Figure 1Bi-v). In contrast, DCC (Deleted in Colorectal Cancer), one of the specific receptors of Netrins [10], was expressed in the GPs regardless of whether the cells were treated with SNP or not (Figure 1Bi, ii).

To investigate the possibility that Netrin signaling might mediate the cell migration and accumulation triggered by NO, RNAi was used to determine the role of Netrins throughout the cell migration and accumulation processes. When Netrin-1 was knocked down by RNAi in GPs (Figure 1Bvi), there were fewer cells with polarized morphology and in migrating chains (Figure 1Bvii) than in the negative and blank controls (Figure 1Bviii, ix). In addition, the number of accumulated cell aggregates decreased by $>50 \%$ in the Netrin- 1 RNAi group in comparison with the control groups $(P<0.05)$, suggesting the GPs migrated more slowly after Netrin-1 knockdown. No significant difference was observed between the test group and control groups after Netrin-4 was knocked down (not shown). Hence, Netrin-1 but not Netrin- 4 was required to mediate the cell movement and accumulation regulated by NO.

To further determine whether Netrin-1 mediated the NO-induced cell migration and accumulation through its specific receptor DCC, DCC blockade was carried out using its antibody (Figure 1C). The results demonstrated that blockade of DCC impaired the GP cell migration and accumulation in a dose-dependent manner (Figure 1C). 
A
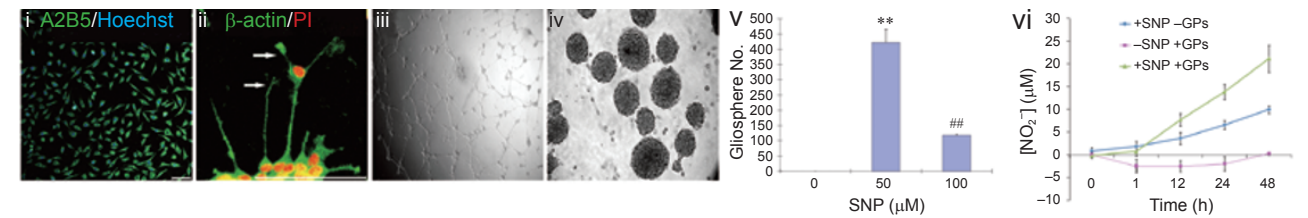

B

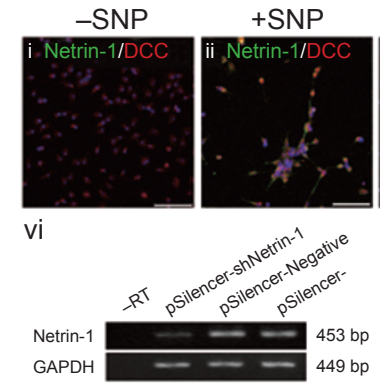

- SNP

+ SNP
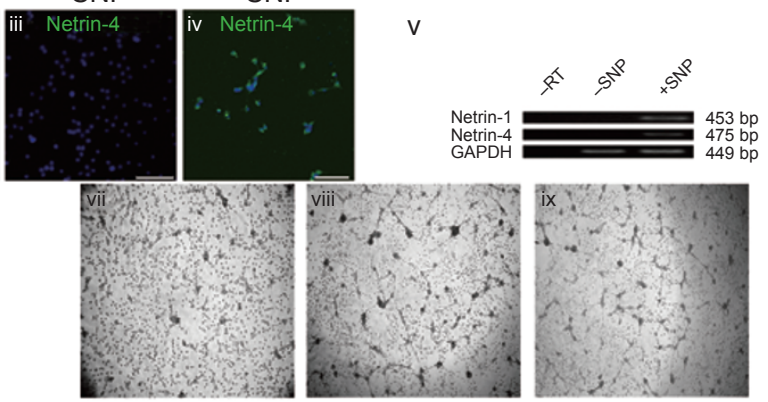

C
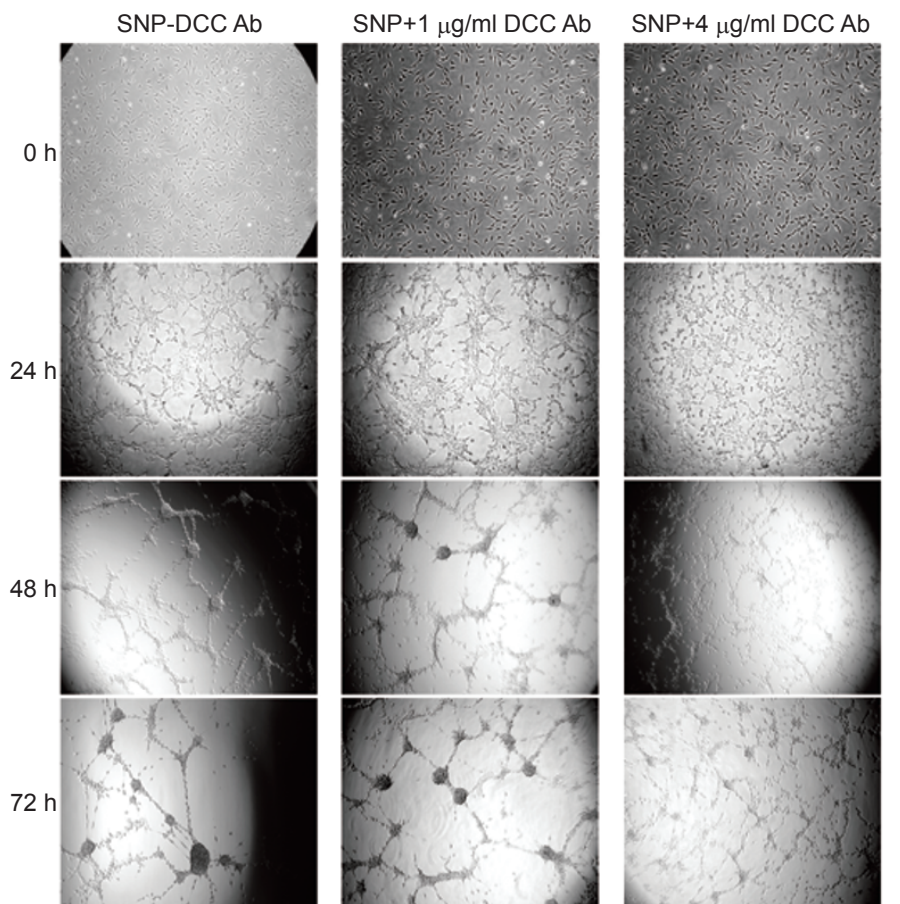

$\mathrm{SNP}+10 \mu \mathrm{g} / \mathrm{ml} \mathrm{DCC}$ Ab
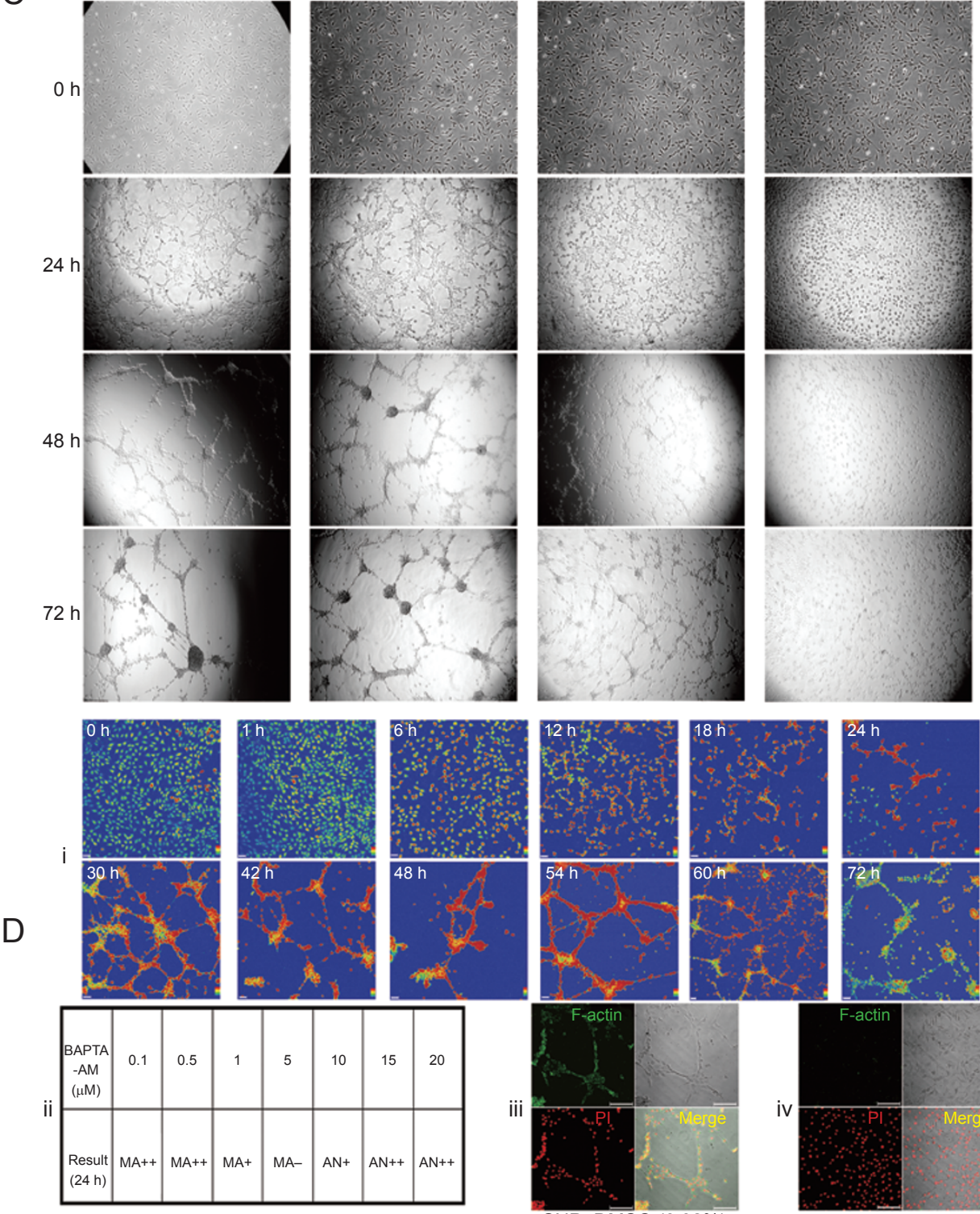

SNP+DMSO $(0.02 \%)$
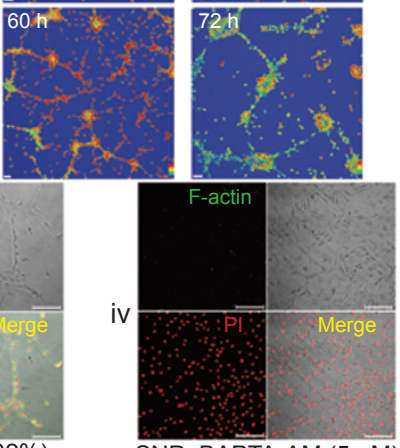
The GPs completely failed to migrate and accumulate when treated with a high concentration of DCC antibody $(10 \mu \mathrm{g} / \mathrm{ml}$, Figure 1C). However, the cell migration and accumulation could be rescued when DCC antibody was washed out (data not shown), suggesting that the DCC receptor was vital to the NO-induced cell migration and aggregation resulting from NO treatment. Therefore, the Netrin-1/DCC pathway is necessary for mediating the NO-induced GP cell migration and accumulation.

Although cyclic nucleotides have been regarded as principal mediators of NO-orchestrated neurite outgrowth, and migration of enteric neurons and glial cells in invertebrates [4], pharmacological changes to intracellular cGMP did not influence the NO-induced GP cell migration and accumulation (data not shown). We therefore examined the changes in intracellular $\mathrm{Ca}^{2+}$, another second messenger, to determine whether it was a downstream regulator in NO signaling. The Flura-3 fluorescence results indicated that after SNP addition, $\left[\mathrm{Ca}^{2+}\right]_{\mathrm{i}}$ gradually increased until gliosphere formation (Figure 1Di). A higher level of $\left[\mathrm{Ca}^{2+}\right]_{i}$ was observed in cells in migrating chains than in non-migratory cells in aggregates (Figure 1Di), suggesting that $\mathrm{Ca}^{2+}$ might be involved in regulating NO-induced cell motility.

To test this hypothesis, intracellular $\mathrm{Ca}^{2+}$ was chelated by its chelator BAPTA-AM. Moderate chelation of intracellular $\mathrm{Ca}^{2+}$ influenced the NO-induced cell migration and accumulation in a dose-dependent manner (Figure 1Dii). The GPs lost their ability to assemble F-actin (Figure 1Div), and failed to migrate and accumulate when treated with SNP and $5 \mu \mathrm{M}$ BAPTA-AM, confirming that
$\mathrm{Ca}^{2+}$ participated in regulating NO-induced cell motility. However, it is still not clear whether intracellular $\mathrm{Ca}^{2+}$ directly mediates the NO signaling before or after Netrin/ DCC signaling as it has been reported to be the downstream element in Netrin/DCC signaling [5]. Excessive $\mathrm{Ca}^{2+}$ chelating caused cell apoptosis/necrosis (Supplementary information, Figure S2), indicating that it was also necessary for regulating other important physiological functions.

In conclusion, we show that NO induces cell migration and accumulation of GPs derived from the rhesus monkey ES cells in vitro. Furthermore, the NO-induced cell migration and accumulation are mediated by $\mathrm{Ne}$ trin-1/DCC signaling, and likely also involve $\mathrm{Ca}^{2+}$. This novel mechanism implicates $\mathrm{NO}$ as a potential significant regulator of neuron-glia interaction, neural network establishment, and even nerve cell repair.

(Experimental materials and methods are depicted in the Supplementary information, Data S1)

\section{Acknowledgments}

We thank Drs Bin Li and Jian Li (Kunming Institute of Zoology) for their help with time-lapse video imaging. This work was supported by research grants from the National Basic Research Program (2006CB701505) and the Chinese Academy of Sciences (KSCX2-YW-R-47).

Hongwei Chen ${ }^{1,2,3}$, Qiang Wei ${ }^{1,2,3}$, Jing Zhang ${ }^{1,2,3}$, Chuankun $\mathrm{Xu}^{4}$, Tao Tan ${ }^{1,2,3}$, Weizhi $\mathrm{Ji}^{1,3}$

${ }^{I}$ Kunming Primate Research Center and Laboratory of Repro-

Figure 1 Netrin-1 signaling mediates NO-induced glial precursor migration and accumulation. (A) NO induced the migration and accumulation of the glial precursors. $\mathrm{A}_{2} \mathrm{~B}^{+}$glial precursors $(\mathrm{i}, 0 \mathrm{~h}$ ) were induced to migrate and accumulate by sodium nitroprusside (SNP, ii-iv). Neuritic outgrowth with abundant $\beta$-actin distribution at the growth cone-like structures (ii, arrow, 24 h), cell polarization, migration in chains (iii, $36 \mathrm{~h}$ ), and eventual accumulation into gliospheres (iv, 72 h) gradually occurred. (v) The relationship between SNP concentration and the number of gliospheres accumulated in $48 \mathrm{~h}$ after SNP treatment $(n=5$ in three independent experiments; **, \#\#, $P<0.01$ ). (vi) $\left[\mathrm{NO}_{2}^{-}\right]$measurement of conditioned media using the Griess method ( $n \geq 6$ in three independent experiments). (B) Netrin-1 mediated the NO-induced cell migration and accumulation. Gene expression analysis by immunostaining (i-iv) and semi-quantitative RT-PCR (v) showed that Netrins (Netrin-1, ii, v; Netrin-4, iv, v) were up-regulated in the glial precursors after SNP treatment compared to the cells without SNP treatment (i, iii, v). DCC, a Netrin-1 receptor, was expressed in the glial precursors both before (i) and after SNP treatment (ii). The glial precursors' capability of migrating and accumulating into gliospheres was impaired by RNAi of Netrin-1 (vi, vii) in contrast to the negative control (viii) and blank control (ix) ( $n=6$ in three independent experiments). (C) Blockade of DCC receptor by its antibody (Ab) inhibited the NO-induced cell migration and accumulation in a dose-dependent way $(n=6$, similar results were observed in another two independent experiments). (D) $\mathrm{Ca}^{2+}$ was involved in regulating the NO-induced cell migration and accumulation. (i) Flura-3 fluorescence showed that after SNP treatment intracellular $\left[\mathrm{Ca}^{2+}\right]_{\mathrm{i}}$ continuously increased to a higher level at early stages $(0-18 \mathrm{~h})$, then was maintained at the higher level accompanied by cell migration and accumulation (24-60 h), and finally decreased at late stages $(72 \mathrm{~h})$, with the majority of the cells in the form of gliospheres. (ii) Chelation of intracellular $\mathrm{Ca}^{2+}$ by BAPTA-AM led to different results in the glial cells treated with SNP ( $n=6$ in three independent experiments). "MA" and "AN" represent "migration and accumulation" and "apoptosis/necrosis", respectively. ++, excellent; +, modest; -, negative. The glial precursors failed to polarize (F-actin ${ }^{-}$, iv), migrate and accumulate when intracellular $\mathrm{Ca}^{2+}$ was chelated by BAPTA-AM $(5 \mu \mathrm{M}$, iv), while the cells in the control group were not influenced (iii). Scale bar: $100 \mu \mathrm{m}$ (Ai-ii; Bi-iv; Di, iii-iv). Magnification: 40× (Aiii; Bvii-ix; C), 100x (Aiv). Nuclei were stained with Hoechst 33258 (Ai; Bi-iv) or PI (Aii; Diii-iv). 
ductive and Developmental Biology, Kunming Institute of Zoology, Chinese Academy of Sciences, Kunming 650223, China; ${ }^{2}$ Graduate University of the Chinese Academy of Sciences, Beijing 100049, China; ${ }^{3}$ Yunnan Key Laboratory of Animal Reproductive Biology, Kunming 650223, China; ${ }^{4}$ Yunnan University, Kunming 650091, China

Correspondence: Weizhi Ji

Tel/Fax: +86-871-5139413

E-mail:wji@mail.kiz.ac.cn

\section{References}

1 Hatten ME. Central nervous system neuronal migration. Annu Rev Neurosci 1999; 22:511-539.

2 Chen A, Kumar SM, Sahley CL, Muller KJ. Nitric oxide influences injury-induced microglial migration and accumulation in the leech CNS. J Neurosci 2000; 20:1036-1043.

3 Knipp S, Bicker G. Regulation of enteric neuron migration by the gaseous messenger molecules $\mathrm{CO}$ and NO. Development
2009; 136:85-93

4 Bicker G. STOP and GO with NO: nitric oxide as a regulator of cell motility in simple brains. Bioessays 2005; 27:495-505.

5 Barallobre MJ, Pascual M, Del Rio JA, Soriano E. The Netrin family of guidance factors: emphasis on Netrin-1 signalling. Brain Res Rev 2005; 49:22-47.

6 Round J, Stein E. Netrin signaling leading to directed growth cone steering. Curr Opin Neurobiol 2007; 17:15-21.

7 Green LC, Wagner DA, Glogowski J, et al. Analysis of nitrate, nitrite, and $[15 \mathrm{~N}]$ nitrate in biological fluids. Anal Biochem 1982; 126:131-138.

8 Jarjour AA, Manitt C, Moore SW, et al. Netrin-1 is a chemorepellent for oligodendrocyte precursor cells in the embryonic spinal cord. J Neurosci 2003; 23:3735-3744.

9 Manitt C, Colicos MA, Thompson KM, et al. Widespread expression of netrin- 1 by neurons and oligodendrocytes in the adult mammalian spinal cord. $J$ Neurosci 2001; 21:3911-3922.

10 Livesey FJ. Netrins and netrin receptors. Cell Mol Life Sci 1999; 56:62-68.

(Supplementary information is linked to the online version of the paper on the Cell Research website.) 